\title{
Bronchorrhoea complicating inflammatory bowel disease
}

A 25 year old Hispanic man with a 3 year history of ulcerative colitis presented with 8 months of productive cough with over $200 \mathrm{ml}$ of yellow sputum, fatigue, dyspnoea, diarrhoea, night sweats, and weight loss. At the time of presentation the patient was on a low dose of mesalamine (400 $\mathrm{mg}$ three times daily) and azathioprine ( $25 \mathrm{mg} /$ day). Examination revealed coarse crackles at the lung bases and a prolonged expiratory phase. The white blood cell count was normal. The chest radiograph showed bilateral reticulonodular infiltrates with acinonodular opacities, and a high resolution CT scan showed diffuse bronchiectasis, centrilobular nodules, and a "tree-in-bud" pattern (fig 1). Obstruction with air trapping was found on pulmonary function tests. Bronchoscopic examination showed diffuse purulent secretions throughout the major airways (fig 2A). BAL fluid smears and cultures were negative for acid-fast bacilli, fungus, and bacterial pathogens. Transbronchial biopsies revealed dense lymphoplasmacytic inflammation and infiltration of the bronchial mucosa (fig 2B). Rapid resolution of pulmonary and gastrointestinal symptoms occurred after initiation of prednisone $40 \mathrm{mg} /$ day and an increase in azathioprine to $100 \mathrm{mg} /$ day.

Pulmonary manifestations of inflammatory bowel disease are uncommon and present as a clinical spectrum including airway disease, interstitial lung disease, parenchymal nodules, and serositis. ${ }^{12}$ Diagnosis is often delayed. ${ }^{3}$ Our patient presented with a chief complaint of bronchorrhoea due to diffuse bronchiectasis and bronchiolitis which ultimately proved to be due to inflammatory bowel disease.

R Jain, D Scheurich

Department of Internal Medicine, University of Texas Southwestern Medical Center, Dallas, TX, USA

G $M$ Lindberg

Department of Pathology, University of Texas Southwestern Medical Center, Dallas, TX, USA

Division of Pulmonary and Critical Care Medicine, University of Texas Southwestern Medical Center, Dallas, TX, USA Correspondence to: Dr C Girod, Division of Pulmonary and Critical Care Medicine, Department of Internal Medicine, University of Texas Southwestern Medical School, Dallas, TX 75390-9034, USA; carlos.girod@utsouthwestern.edu

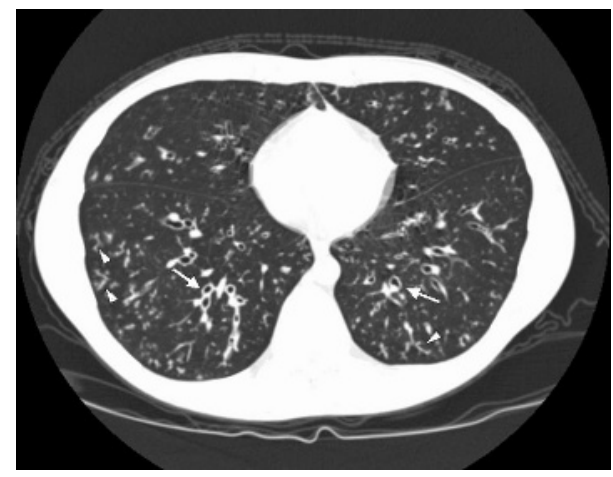

Figure 1 High resolution CT scan showing diffuse lower lobe cylindrical bronchiectasis (arrows) and centrilobular nodules with a "tree-in-bud" appearance (arrowheads).
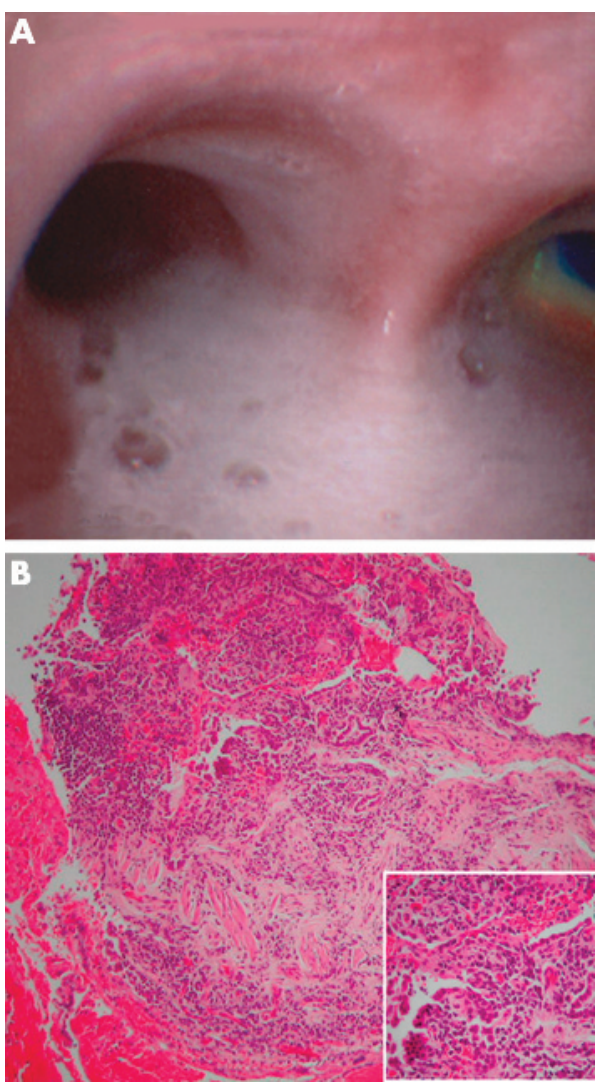

Figure 2 (A) Bronchoscopy showing purulent secretions. (B) Low power photomicrograph showing distortion of the bronchial wall with transmural chronic inflammation and fibrosis. Inset shows plasma cellrich inflammation involving the bronchial mucosa. No granulomas were seen.

\section{Learning points}

- Pulmonary manifestations of inflammatory bowel disease are rare and have a variety of clinical and radiographic presentations that primarily include bronchiectasis, chronic bronchitis, and bronchiolitis.

- After thoroughly excluding infections and other aetiologies of pulmonary symptoms in patients with inflammatory bowel disease, immunomodulating therapy should be started promptly, given the dramatic clinical response.

\section{REFERENCES}

1 Eaton TE, Lambie N, Well AU. Bronchiectasis following colectomy for Crohn's disease. Thorax 1998;53:529-31.

2 Camus P, Colby TV. The lung in inflammatory bowel disease. Eur Respir J 2000;15:5-10.

3 Mahadeva R, Walsh G, Flower CD, et al. Clinical and radiological characteristics of lung disease in inflammatory bowel disease. Eur Respir $J$ 2000;15:41-8 